\title{
Quasars and their enviroments along cosmic history
}

\author{
Tiziana Di Matteo ${ }^{1}$, Rupert A.C. Croft ${ }^{2}$, Volker Springel ${ }^{1}$ \\ and Lars Henrquist ${ }^{3}$ \\ ${ }^{1}$ Max-Planck-Institut für Astrophysik, Karl-Schwarzschild-Str. 1, 85740 Garching bei \\ München, Germany email: tiziana, volker@mpa-garching.mpg.de \\ ${ }^{2}$ Carnegie-Mellon University, Dept. of Physics, 5000 Forbes Ave., Pittsburgh, PA 15213, USA \\ email: rcroft@cmu.edu \\ 3 Harvard-Smithsonian Center for Astrophysics, 60 Garden St., Cambridge, MA 02138, USA \\ email: lhernquist@cfa.harvard.edu
}

\begin{abstract}
The observed properties of supermassive black holes suggest a fundamental link between their assembly and the formation of their host spheroids. We model the growth and activity of black holes in galaxies using $\Lambda \mathrm{CDM}$ cosmological hydrodynamic simulations. We study the evolution of the metal enrichment in quasar hosts and hence explore the relationship between star/spheroid formation and black hole growth/activity in galaxies. Using the simulations we approach the epoch of the first quasars and the first significant star formation activity. We show that the hosts of the rare bright quasars at $z \sim 5-6$ have star formation rates of several hundred $\mathrm{M}_{\odot} \mathrm{yr}^{-1}$ and halo masses of order $\sim 10^{12} \mathrm{M}_{\odot}$. Already at these redshifts they have supersolar $\left(Z / Z_{\odot} \sim 2-3\right)$ central metallicities, with a mild dependence of metallicity on luminosity, consistent with observed trends.
\end{abstract}

\section{Introduction}

The presence of supermassive black holes in the centers of nearby galaxies with a significant spheroidal component supports arguments that there is a fundamental link between the assembly of black holes and the formation of spheroids in galaxy halos. The evidence indicates that the mass of the central black hole is correlated with the bulge luminosity (e.g., Magorrian et al. 1998) and even more tightly with the velocity dispersion of its host bulge (e.g.; Ferrarese \& Merritt 2000; Gebhardt et al. 2000). We briefly describe a prescription for the growth and activity of black holes in $\Lambda$ CDM cosmological hydrodynamic simulations (see Di Matteo et al. 2003a,b for details) and how this can be used to study the properties of quasar hosts up to high redshifts. In particular, we will concentrate on studying the chemical enrichment of quasar host galaxies. Quasar metallicities offer us a potentially powerful probe of the Universe at redshifts beyond those at which objects have been detected so far. The star formation timescales necessary to build up a reservoir of metal-rich gas by $z \sim 4-5$ indicate the presence of massive star formation already at $z \sim 6-10$. Other than the possible signature of reionization at $z \sim 17-20$ seen by the WMAP satellite (Kogut et al. 2003), information on the Universe at these epochs is difficult to obtain.

\section{Simulations and analysis}

Throughout, we shall use a set of cosmological simulations for a $\Lambda$ CDM model, with $\Omega_{\Lambda}=0.7, \Omega_{\mathrm{m}}=0.3$, baryon density $\Omega_{\mathrm{b}}=0.04$, a Hubble constant $H_{0}=100 h \mathrm{kms}^{-1} \mathrm{Mpc}^{-1}$ 
(with $h=0.7$ ). The simulations include a novel prescription for star formation and feedback processes in the interstellar medium that is able to yield a numerically converged prediction for the full star formation history. Here, we refer the reader to Springel \& Hernquist $(2003 \mathrm{a}, \mathrm{b})$ for a full description of simulations. The simulations keep track of metal enrichment and the dynamical transport of metals by the motion of gas particles. Metals are produced by stars which enrich the gas by supernova explosions. The mass of metals returned to the gas is $\Delta M_{Z}=y_{*} \Delta M_{*}$, where $y_{*}=0.02$ is the yield, and $\Delta M_{*}$ is the mass of newly formed stars. Assuming that metals are being instantaneously mixed with the cold clouds and the ambient hot gas, the metallicity $Z=M_{Z} / M_{g}$ of a star-forming gas particle increases during one timestep $\Delta t$ in the simulations by

$$
\Delta Z=(1-\beta) y_{*} \frac{\rho_{c}}{\rho} \frac{\Delta t}{t_{*}},
$$

where $\rho_{c} / \rho$ is the mass fraction of the gas in the cold phase, $\beta$ is the mass fraction of stars that are short lived and instantly turn into supernovae, and $t_{*}$ is the star formation timescale, where the value $t_{0}^{*}=2.1 \mathrm{Gyr}$ is chosen to match the Kennicutt law (1998).

\subsection{Quasar model}

In order to select quasar hosts from the simulations and determine the quasar properties from the simulations (in which black holes have not been self-consistently included) we make use of the model described in Di Matteo et al. (2003). Here we briefly summarize the main characteristics of this scheme. The model starts from the hypothesis that black holes grow and shine by gas accretion, the supply of which is regulated by the interplay with star formation in spheroids. The black hole mass growth saturates in response to star formation and its associated feedback processes. This results in black holes masses that are related to the velocity dispersion of their host spheroids in a manner which is consistent with the observed $M-\sigma$ relation (see Fig. 3 in Di Matteo et al. 2003). The QSO luminosity function evolves as a result of the declining amount of fuel available for accretion (and star formation), assuming a given quasar lifetime and duty cycle. Within the context of this simple model the total black hole accretion rate density closely tracks the star formation rate density, as expected if black hole growth and fueling are fundamentally linked to the assembly of the spheroids and their star formation rate, respectively. All galaxies are assumed to undergo active phases with a duty cycle given simply by $f_{Q}=t_{Q} / t_{H(z)}$, where $t_{Q}$ is the quasar lifetime and is the only free parameter of the model. In order to estimate the luminosity of each quasar, we simply assume that all the gas in the galaxy is in principle available for accretion on this timescale. The bolometric luminosity owing to accretion onto the central black hole at redshift $z$ is then given by

$$
L=\eta \dot{M}(z) c^{2} \sim \eta \frac{\Delta M_{\mathrm{gas}}}{\Delta t} c^{2} \sim \eta f M_{\mathrm{gas}}(z) c^{2} / t_{Q}
$$

where we have taken $\Delta M_{\text {gas }}(z) \sim f M_{\text {gas }}(z)$, assuming that the average mass accreted is a constant fraction $f$ of the total gas mass. We adopt the standard value for accretion radiative efficiency of $\eta=10 \%$. With this model and $t_{Q} \sim 2-4 \times 10^{7} \mathrm{yr}$, we are able to reproduce the observed quasar luminosity function reasonably well, particularly for $z>2$. Figure 1 shows the evolution of the comoving number of bright quasars in the Bband (with absolute magnitude $m_{B}<-26$ ) and in the X-rays compared to observations. A general feature of this model is that the black hole accretion rate history is found to closely follow the cosmic star formation rate density, therefore establishing a direct relation between black hole activity and star formation. A consequence of this is that at high redshifts we expect the evolution of black hole activity to be mostly driven by the 

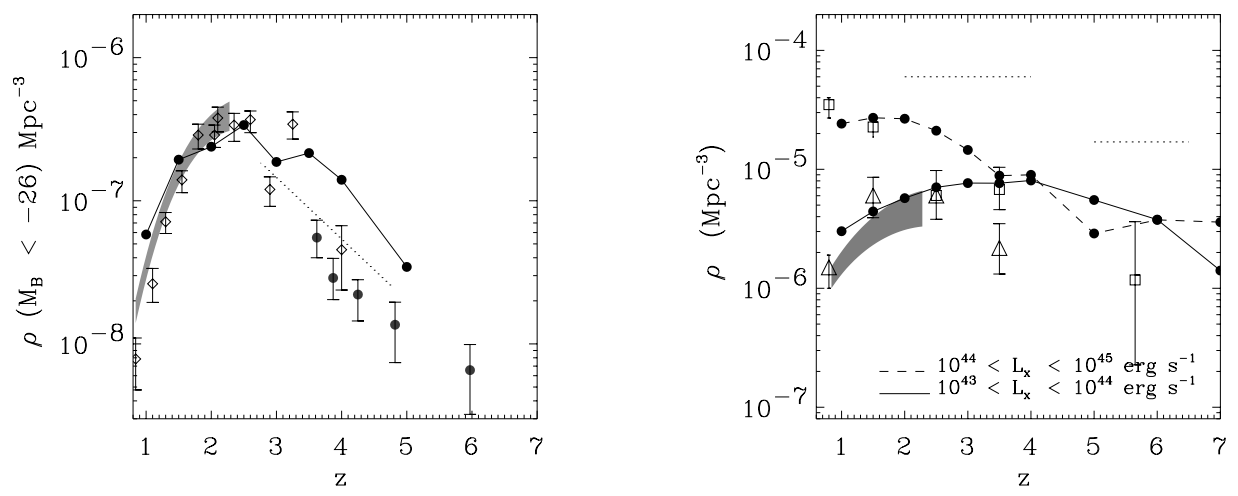

Figure 1. Left: The evolution of the space density of quasars with $M_{\mathrm{B}}<-26$. Our model is shown by the solid circles joined by the solid lines. The model is for $t_{Q}=2 \times 10^{7} \mathrm{yr}$. The dotted line is a fit to the SSG survey. The open squares show the compilation from Pei (1995) derived from the WHO and HS surveys. The filled circles are the SDSS points derived by Fan et al. (2001a,b). The shaded area is the best fit model from the 2dF by Boyle et al. (2000). Right: The evolution of space density of quasar in the $2-8 \mathrm{keV}$ X-ray energy band. The data are from Chandra observations by Cowie et al. (2003); Barger et al. (2003). The solid and dashed lines show our model.

$\begin{array}{cccc}\text { Redshift } & \begin{array}{c}M_{\mathrm{DM}} \\ 10^{12} h^{-1} \mathrm{M}_{\odot}\end{array} & \begin{array}{c}M_{\mathrm{BH}} \\ 10^{9} h^{-1} \mathrm{M}_{\odot}\end{array} & \begin{array}{c}S F R \\ \mathrm{M}_{\odot} \mathrm{yr}^{-1}\end{array} \\ 2 & 3 & 1.3 & 38 \\ 5 & 1.8 & 0.9 & 600 \\ 6.5 & 1 & 0.8 & 270\end{array}$

Table 1. The dark matter, black hole masses and star formation rates for the objects in Figures 2 and 3 .

gravitational growth of structure, as this is the case for the star formation rate density relatively independent of the details of the gas dynamics.

\section{The quasar hosts}

Using the simulations, we can predict the properties of quasar host galaxies at high redshifts. In our model, bright rare quasars at $z>5$ reside in the rare, most massive halos which are already forming stars vigorously at these times (see Table 1).

Most of the black hole growth occurs simultaneously with the processes that dump matter into the central regions of galaxies and induce substantial star formation. In particular, for our black hole growth model, we find typical black hole masses for these objects of order $M_{\mathrm{BH}} \sim 10^{9} \mathrm{M}_{\odot}$. The quasar phase at high redshift follows or is roughly co-eval with the major star formation event in the most massive objects. Using simulations we have measured the distribution of the gas metallicity and density in quasar host galaxies from $z=8$ to $z=0$. Typically there is a strong radial gradient in the metallicity of the gas in halos, with the central densest regions of galaxies reaching up to a few times the solar metallicity while the gas in the outer regions has a metallicity which is at most around a few tenths of solar (Di Matteo et al. 2004). 

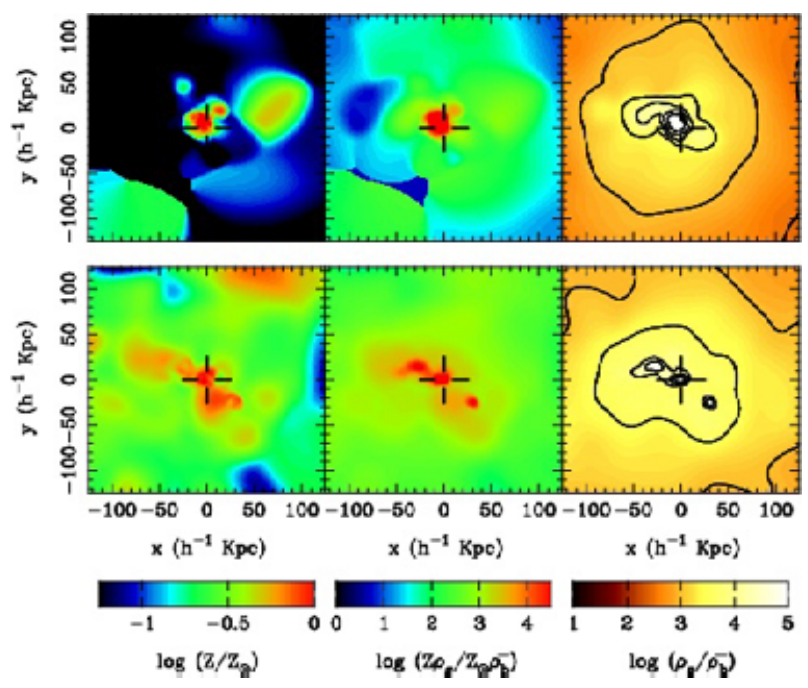

Figure 2. Two quasar hosts in the G5 simulation, one at $z=5$ (top row) and one at $z=2$ (bottom row). For each host, we show from left to right the metallicity, the projected metal density (proportional to the mass of metals), and the gas density. The panels extend $250 h^{-1} \mathrm{kpc}$ in the $x$ and $y$ direction and $100 h^{-1} \mathrm{kpc}$ in the $z$-axis. and are centered on the quasars (positions shown by crosshairs). The quasars have magnitude $m_{B}=-26.3$ (top) and $m_{B}=-26.7$ (bottom), and the corresponding masses of their host halos are $1.8 \times 10^{12} h^{-1} \mathrm{M}_{\odot}$ and $3.1 \times 10^{12} h^{-1} \mathrm{M}_{\odot}$, respectively.

Visualizing the morphology of the metal-rich gas in and around quasar hosts can tell us directly the properties of the hosts themselves and how the metal enrichment occurs in these high density loci and how uniformly the metals are distributed in response to star formation and its associated feedback. In Figure 2, we show a plot of the hosts of two typical bright quasars (with $B$-band magnitude $m_{\mathrm{b}}<-26$ ), which were picked randomly from the $z=5$ and the $z=2$ outputs of the G5 simulation. In Table 1 , we summarize some of the main properties of these hosts, such as their dark matter, stellar and black hole masses together with their central star formation rates. In both cases, the metallicity close to the center (indicated by the cross on the figure) is around solar, and the mass of their host halos is similar and of the order of a few $10^{12} h^{-1} \mathrm{M}_{\odot}$. The $z=5$ quasar rests in a more concentrated island of metal rich gas than the $z=2$ case, where the edges of the central enhancement are more diffuse. It is evident, therefore, that although the mean metallicity of the ISM is lower at $z=5$, the central regions do not show significant evolution. We find that in the central regions, star formation, and consequently metal enrichment proceeds rather quickly because of the high densities entailing short star formation timescales (Eq. 2). This plot also shows that star formation occurs in islands of high gas density that lie nearby in the $z=2$ case, so that the high metallicity of the gas 50 to $100 h^{-1} \mathrm{kpc}$ away from the quasar has likely been generated in situ rather than being blown there by winds.

As well as examining the central region of different bright quasar hosts active at different redshifts, we can also look at the history of gas that was once at the center of an early quasar. In this way, we can see how the large scale movements of hosts through the evolving density field impact their metallicity and study the relationship between the quasar phase and the first major star formation event. In Figure 3, we follow the position of what was initially the most tightly bound particle in one of the earliest bright quasars to be active in the G5 simulation, at $z=6.5$. We can see that at $z=6.5$, the metallicity 

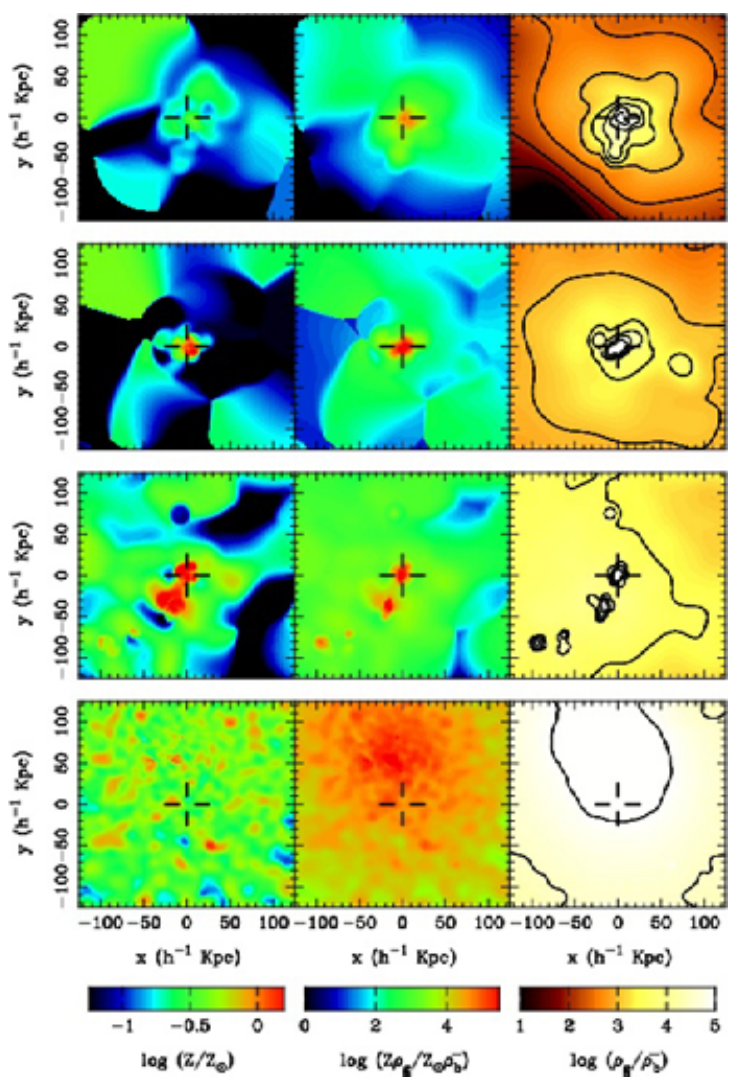

Figure 3. Time evolution of a gas element initially identified in a quasar host at $z=6.5$. We show metallicity (left panels), projected metal density (center panels), and projected gas density (right panels), for slices of $100 h^{-1} \mathrm{kpc}$ width in the $z$-direction and and $250 \mathrm{~h}^{-1} \mathrm{kpc}$ in $x$ and $y$ (same as Fig. 2). The top row of panels shows the situation at $z=6.5$, centered on the quasar position. At this time the quasar has absolute magnitude $m_{B}=-26.2$ and resides in a halo of mass $1.1 \times 10^{12} \mathrm{M}_{\odot}$. In the succeeding three rows, each panel is centered on the position of the particle which was the most bound at $z=6.5$ (closest to the quasar), but at the later redshifts of $z=4, z=2$, and $z=0$.

near the center of the host is well below solar, although the mass of the halo is relatively high, of order $\sim 10^{12} h^{-1} \mathrm{M}_{\odot}$. Because in our model the quasar phase is dependent only on the amount of gas in the halo, it can, at these early times, be coeval with the first major event of star formation in the galaxy. We note that the star formation rate in the galaxy measured in the simulation corresponds to $\sim 200 \mathrm{M}_{\odot} \mathrm{yr}^{-1}$ (see Table 1 ). The metallicity builds up rapidly to values of around solar in the central region, which can be seen in the $z=4$ timeslice. By $z=5$, we find that the mass weighted average metallicity of gas particles within $10 h^{-1} \mathrm{kpc}$ of the central particle is 0.8 solar. Outside $\sim 20 h^{-1} \mathrm{kpc}$ not much has changed, however.

Finally, we compare the results from the simulations (Fig. 4) with the observed values of gas metallicity estimated from the broad emission line region (BELR) of bright quasars (e.g. Dietrich et al. 2003a,b). The simulations suggest that the measured supersolar metallicities of quasars are representative of the highest density gas that resides in the innermost regions of galaxies which undergoes the fastest modes of star formation and chemical enrichment. The simulations also predict little or no evolution of the mean quasar metallicity in the measured redshift range of $3.5<z<5$. The high, 


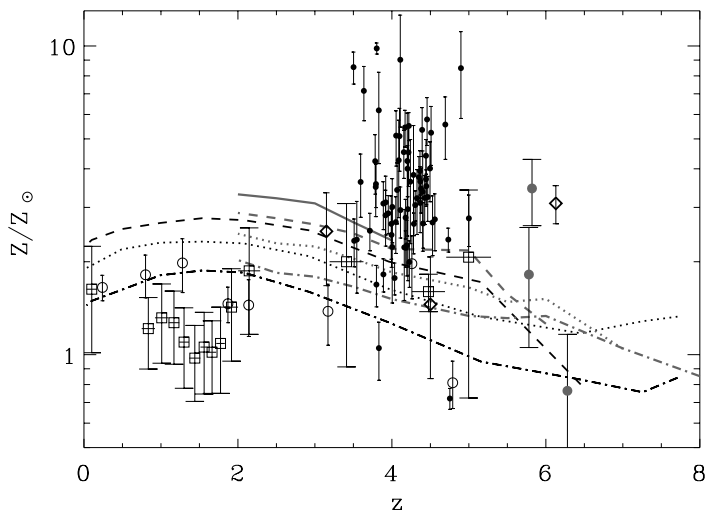

Figure 4. Evolution of the mean gas metallicities (top panel) and standard deviations (bottom panel) of QSOs with $m_{b}<-26$ as a function of redshift. The results from the G5 and G6 simulation runs (see Di Matteo et al. 2003b) are shown with solid, dashed, dotted, dash-dotted lines for quasar lifetimes $t_{\mathrm{Q}}=4,2,1,0.5 \times 10^{7} \mathrm{yr}$, respectively. The data is from Dietrich et al. 2003a,b., Freudling et al. 2003.; Hiwamura et al. 2000

solar/super-solar-like metallicities at high redshifts are achieved quickly in rare and isolated islands of high-density gas residing in the first halos that form. They thus indicate the presence of massive star formation already at $z \sim 6-7$ (see Table 1 and Di Matteo et al. 2004).

\section{Conclusions}

We have used cosmological hydrodynamical simulations coupled with a prescription for black hole activity in galaxies to study the evolution of the metal enrichment in quasar host galaxies and explore the relation between star/spheroid formation and black hole growth/activity. We compared the results from the simulations with the observed values of gas metallicity estimated from the broad emission line region of bright quasars (e.g. Dietrich et al. 2003a,b). The simulations suggest that the super-solar metallicities of quasars deduced from these studies are representative of the highest density gas that resides in the innermost regions of galaxies. $\mathrm{n}$ this picture, the very high metallicities probed by the BELR are found only in a very small fraction of the gas, within the nuclear regions that undergoes the fastest modes of star formation and chemical enrichment. In other words, the self-regulated, quiescent mode of star formation implemented in the simulations can account well for the mildly super-solar metallicity of the gas seen around bright quasars without any need for strong bursts or top heavy IMF.

\section{References}

Cowie, L. L., et al. 2003, ApJ, in press

Dietrich, M., et al. 2003a, A\&A, 398, 891

Dietrich, M., et al. 2003b, ApJ, 589, 722

Di Matteo, T., Croft, R. A. C., Springel, V., Hernquist, L. 2003a, ApJ, 593, 56

Di Matteo, T., et al. 2004, ApJ, in press

Gebhardt, K., et al. 2000, ApJ, 539, L13

Fan, X. et al. 2001b, ApJ, 122, 2833

Ferrarese, L., \& Merritt, D. 2000, ApJ, 539, L9

Springel, V., \& Hernquist, L. 2003, MNRAS, 339, 312 